\title{
Adubação fosfatada para cultivares de mamoneira no Rio Grande do Sul
}

\author{
Phosphate fertilization to castor bean cultivars in Rio Grande do Sul State, Brazil
}

\author{
Jaqueline Pereira Machado de OliveiraI Walkyria Bueno Scivittaro ${ }^{\text {II }}$

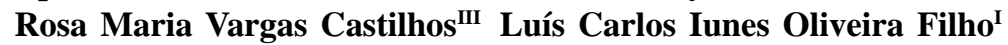

\begin{abstract}
- NOTA -
RESUMO

O estudo foi realizado na safra agrícola 2007/08, em um Planossolo Háplico, em Pelotas, Rio Grande do Sul (RS). Os tratamentos compreenderam as cultivares de mamoneira 'AL Guarany 2002' e 'Lyra', com cinco doses de $P\left(0 ; 30 ; 60 ; 90\right.$ e $120 \mathrm{~kg} \mathrm{ha}^{-1}$ de $\mathrm{P}_{2} \mathrm{O}_{5}$ ), sendo dispostas em delineamento blocos ao acaso, em esquema fatorial, com três repetições. Avaliaram-se o estado nutricional e o desempenho produtivo da cultura. A adubação fosfatada alterou os teores

castor bean foliar concentrations of nitrogen, calcium, boron, and iron. For boron, this effect was limited to the 'Lyra' cultivar. The 'AL Guarany 2002' cultivar demanded more phophorus than 'Lyra' cultivar. Phosphate fertilization increased grain yield of both castor bean cultivars. Yield increased with the use of up to $156 \mathrm{~kg} \mathrm{ha}^{-1}$ of $\mathrm{P}_{2} \mathrm{O}_{5}$, for 'AL Guarany 2002' cultivar, and of up to $90 \mathrm{~kg} \mathrm{ha}^{-1}$ of $\mathrm{P}_{2} \mathrm{O}_{5}$, for 'Lyra' cultivar. The rates of maximum economic efficiency for 'AL Guarany 2002' and 'Lyra' cultivars were, respectively, $134 \mathrm{~kg} \mathrm{ha}^{-1}$ and $78 \mathrm{~kg} \mathrm{ha}^{-1}$ of $\mathrm{P}_{2} \mathrm{O}_{5}$.
\end{abstract} foliares de $\mathrm{N}, \mathrm{Ca}, \mathrm{B}$ e Fe da mamoneira; para boro, o efeito restringiu-se à 'Lyra'. A cultivar 'AL Guarany 2002' mostrouse mais exigente em fósforo que a 'Lyra'. A adubação fosfatada elevou a produtividade de grãos de ambas as cultivares de mamoneira. Esta aumentou com o uso de até $156 \mathrm{~kg} \mathrm{ha}^{-1}$ de $\mathrm{P}_{2} \mathrm{O}_{5}$, para a 'AL Guarany 2002', e de até $90 \mathrm{~kg} \mathrm{ha}^{-1}$ de $\mathrm{P}_{2} \mathrm{O}_{5}$ para a 'Lyra', e as doses de máxima eficiência econômica para essas cultivares foram, respectivamente, de $134 \mathrm{~kg} \mathrm{ha}^{-1} e$ $78 \mathrm{~kg} \mathrm{ha}^{-1}$ de $\mathrm{P}_{2} \mathrm{O}_{5}$.

Palavras-chave: Ricinus communis, fósforo, adubo, estado nutricional, produtividade da mamona.

\section{ABSTRACT}

The study was carried out from April 2007 to May 2008, on a Planossolo Háplico (Albaqualf), in Pelotas, RS, Brazil. The treatments comprised the castor bean cultivars ' $\mathrm{AL}$ Guarany 2002' and 'Lyra', with five rates of phosphate ( 0 ; 30; 60; 90 and $120 \mathrm{~kg} \mathrm{ha}^{-1}$ of $\left.\mathrm{P}_{2} \mathrm{O}_{5}\right)$. These were arranged as a randomized complete block in a factorial design with three replications. Castor bean nutritional status and yield performance were evaluated. The phosphate fertilization altered
Key words: Ricinus communis, phosphorus, fertilizer, nutritional status, castor bean grain yield.

Nos últimos anos, o cultivo da mamona tornou-se um agronegócio promissor na região Sul do Brasil, devido ao potencial da cultura para a produção de biodiesel. O Estado do Rio Grande do Sul tem recebido incentivos governamentais para expandir o cultivo, estando o sucesso dessa iniciativa condicionado à geração de tecnologias de produção da matéria-prima, incluindo o estabelecimento de recomendações de adubação regionalizadas. A mamoneira contém teores elevados de óleo e proteínas nas sementes, demandando quantidades elevadas de nutrientes, especialmente nitrogênio, fósforo, potássio, cálcio e magnésio (SAVY FILHO, 2005). Dessa forma, a obtenção de produtividades elevadas requer o cultivo em solos férteis ou suplementados via adubação.

IPrograma de Pós-graduação em Agronomia, Faculdade de Agronomia Eliseu Maciel (FAEM), Universidade Federal de Pelotas (UFPel), Pelotas, RS, Brasil.

IIEmbrapa Clima Temperado, 96001-970, Pelotas, RS, Brasil. E-mail: walkyria.scivittaro@cpact.embrapa.br. Autor para correspondência.

IIIDepartamento de Solos, FAEM, UFPel, Pelotas, RS, Brasil. 
Entre os nutrientes utilizados na adubação da mamoneira, o fósforo destaca-se como o elemento mais estudado e com maior resposta em produtividade (SOUZA et al., 1974; NAKAGAWA et al., 1986; LAVRES JUNIOR et al., 2009), o que está associado, principalmente, à baixa eficiência de absorção do nutriente pela cultura (SOUZA et al., 1985). O nutriente é essencial ao crescimento da planta, que consome grande quantidade de energia para garantir o armazenamento de óleo nas sementes (FERREIRA et al., 2004). Pela carência de resultados de pesquisa locais, realizou-se um experimento para avaliar a resposta das cultivares de mamona 'AL Guarany 2002' e 'Lyra' à adubação fosfatada nas condições edafoclimáticas da região Sul do Rio Grande do Sul.

O experimento foi desenvolvido na safra 2007/08, em área de produção comercial, no município de Pelotas, Rio Grande do Sul (RS), em um Planossolo Háplico (SANTOS et al., 2006), com teor inicial de fósforo baixo (5,9mg dm ${ }^{-3}$ ) (CQFS-RS/SC, 2004). Utilizaram-se as cultivares de mamona 'AL Guarany 2002', de porte médio, frutos indeiscentes e ciclo de aproximadamente 180 dias até a colheita de cachos terciários, e o híbrido 'Lyra', de porte baixo, frutos indeiscentes e ciclo precoce (SAVY FILHO, 2005). Para cada cultivar, avaliaram-se cinco doses de fósforo (0; 30; 60; 90 e $120 \mathrm{~kg} \mathrm{ha}^{-1} \mathrm{de}_{2} \mathrm{O}_{5}$ ), na forma de superfosfato triplo.

Os tratamentos foram delineados em blocos ao acaso, em esquema fatorial $2 \times 5$, com três repetições. As dimensões das parcelas experimentais foram: 6,4mx8m, para a ‘AL Guarany 2002', e 3,2mx8m, para a 'Lyra', em razão de variações no espaçamento entre linhas. Para a 'AL Guarany 2002', este foi de 1,6mx0,8m e para 'Lyra', de $0,8 \mathrm{mx} 0,8 \mathrm{~m}$. As parcelas experimentais foram constituídas por quatro linhas de planta, sendo consideradas na parcela útil apenas as duas centrais. A cultura foi implantada em sistema convencional de preparo do solo, e a semeadura foi realizada manualmente, mantendo-se uma planta por cova, após desbaste. O fertilizante fosfatado foi aplicado integralmente na semeadura. Nessa ocasião, aplicaramse, ainda, $30 \mathrm{~kg} \mathrm{ha}^{-1}$ de $\mathrm{N}$ (ureia) e $60 \mathrm{~kg} \mathrm{ha}^{-1}$ de $\mathrm{K}_{2} \mathrm{O}$ (cloreto de potássio). Após 35 dias da emergência, procedeu-se à adubação em cobertura, com $60 \mathrm{~kg} \mathrm{ha}^{-1}$ de $\mathrm{N}$ e $30 \mathrm{~kg} \mathrm{ha}^{-1}$ de $\mathrm{K}_{2} \mathrm{O}$.

Por ocasião da emissão do primeiro cacho, avaliou-se o estado nutricional da mamoneira, coletando-se a quarta folha a partir do ponteiro de 12 plantas por parcela. Para a análise química, descartouse a nervura central das folhas (MALAVOLTA et al., 1997). O material vegetal foi preparado e submetido a análises químicas, sendo determinados os teores de macro e micronutrientes (FREIRE, 2001). A colheita de cachos maduros foi realizada em três etapas. Os frutos foram secos e descascados para a determinação da produtividade de grãos. Com base nesses dados, foi calculada a dose de máxima eficiência econômica, a partir da equação de Mitscherlich, e foram considerados os custos unitários do nutriente (R\$ $4,64 \mathrm{~kg}^{-1}$ de $\mathrm{P}_{2} \mathrm{O}_{5}$ ) e do produto ( $\mathrm{R} \$ 1,33 \mathrm{~kg}^{-1}$ de grão) vigentes no mês de novembro de 2008.

Os dados obtidos foram submetidos à análise de variância, sendo comparadas as médias de cultivares de mamoneira, pelo teste de Duncan (5\%), e comparado o efeito das doses de fósforo, por análise de regressão polinomial (5\%).

Os teores de fósforo, magnésio, manganês e zinco na folha não sofreram influência dos tratamentos, apresentando valores médios de: $4,2 \mathrm{~g} \mathrm{~kg}^{-1}$ de P; 2,8g kg-1 de Mg; $141 \mathrm{mg} \mathrm{kg}^{-1}$ de Mn e 42mg kg-1 de $\mathrm{Zn}$. O teor foliar de $\mathrm{P}$ foi pouco superior à faixa de suficiência indicada para a cultura de $3-4 \mathrm{~g} \mathrm{~kg}^{-1}$ (MALAVOLTA et al., 1997), revelando boa capacidade de absorção do nutriente de ambas as cultivares utilizadas, visto que, independentemente da dose do nutriente e apesar do teor inicial baixo no solo, estas mantiveram teores elevados de $\mathrm{P}$ no tecido. Para Mg, o teor foliar médio foi superior ao nível crítico estabelecido para a cultura (2,5 $\left.\mathrm{g} \mathrm{kg}^{-1}\right)$ (MALAVOLTA et al., 1997). Para Mn e Zn, embora não se disponha na literatura indicação de faixas de suficiência, a comparação dos teores foliares médios desses micronutrientes encontrados com os dados disponíveis na literatura (LÉLES, 2008; PAULO et al., 1989; SOUZA\& NATALE, 1997) indica semelhança de valores.

Os teores foliares de nitrogênio $\left(54,9 \mathrm{~g} \mathrm{~kg}^{-1}\right)$, potássio (22,7 $\left.\mathrm{g} \mathrm{kg}^{-1}\right)$, cálcio $\left(13,6 \mathrm{~g} \mathrm{~kg}{ }^{-1}\right)$, enxofre $(4,3 \mathrm{~g}$ $\mathrm{kg}^{-1}$ ) e cobre (15mg kg-1) da cultivar 'AL Guarany 2002' foram superiores aos da cultivar 'Lyra', respectivamente, $52,7 \mathrm{~g} \mathrm{~kg}^{-1}$ de $\mathrm{N}$; 20,1 $\mathrm{g} \mathrm{kg}^{-1}$ de K; 11,3g $\mathrm{kg}^{-1}$ de Ca; $4,1 \mathrm{~g} \mathrm{~kg}^{-1}$ de $\mathrm{S}$; e $10 \mathrm{mg} \mathrm{kg}^{-1}$ de $\mathrm{Cu}$, demonstrando maior capacidade de absorção de nutrientes da 'AL Guarany 2002', visto que seu porte maior favorece a diluição dos teores de nutrientes no tecido vegetal. Segundo NAKAGAWA \& NEPTUNE (1971), aos 64 dias após a germinação, os níveis críticos de $\mathrm{N}$ e K na folha da mamoneira são, respectivamente, de $41 \mathrm{~g} \mathrm{~kg}^{-1}$ e $46 \mathrm{~g} \mathrm{~kg}^{-1}$. Assim, os teores de $\mathrm{N}$ determinados para ambas as cultivares foram elevados, e os de K, baixos. Considerando os padrões de referência de MALAVOLTA et al. (1997), o comportamento para $\mathrm{N}$ e $\mathrm{K}$ foi semelhante, enquanto os teores de cálcio não atingiram o nível crítico preconizado (15g kg-1) e, contrariamente, os teores foliares de enxofre superaram a faixa de suficiência (2,5- 
3,5g $\left.\mathrm{kg}^{-1}\right)$. Esses padrões foram estabelecidos para cultivares mais antigas, cuja exigência nutricional pode ser distinta das usadas no presente estudo. O teor foliar de cobre da ‘AL Guarany 2002' foi 50\% superior ao da cultivar 'Lyra'; ambas as cultivares superaram o nível de suficiência (7mg kg-1) proposto por LÉLES (2008).

A variação na dose de fósforo influenciou o teor foliar de nitrogênio na mamoneira, ajustando-se a um modelo quadrático com máximo correspondente à dose de $56 \mathrm{~kg} \mathrm{ha}^{-1}$ de $\mathrm{P}_{2} \mathrm{O}_{5}$ (Figura 1a). JESCHKE et al. (1997) avaliaram a interação entre N e P na mamoneira, sendo observada elevação no teor de nitrato na planta, em resposta ao aumento na concentração de $\mathrm{P}$ em solução. Isso se deve ao aumento na eficiência na absorção de ânions pelas plantas com o suprimento adequado de $\mathrm{P}$, uma vez que esse transporte é efetuado contra um gradiente de potencial eletroquímico, requerendo energia na forma de ATP (MALAVOLTA et al., 1997). O teor foliar de cálcio na mamoneira decresceu proporcionalmente ao aumento da dose de $\mathrm{P}$ (Figura 1b), possivelmente em razão da ocorrência de efeito de diluição, decorrente do maior crescimento da planta quando mais adubada (dados não apresentados). Independentemente da dose de fósforo, o teor foliar de boro no híbrido 'Lyra' foi superior ao da 'AL Guarany 2002'. Ademais, apenas a 'Lyra’ sofreu influência da adubação fosfatada; os dados se ajustaram a um modelo linear decrescente (Figura 1c), indicando, para essa cultivar, que a variação no teor foliar de B acompanhou as variações no porte da planta afetado pela dose de P. Para ambas as cultivares, os teores foliares de boro ficaram dentro da faixa de suficiência indicada por SOUZA \& NATALE (1997), de 35 a $91 \mathrm{mg} \mathrm{kg}^{-1}$.

O teor de ferro no tecido foliar da mamoneira variou com a dose de fósforo (Figura 1d), sendo ajustado um modelo quadrático com valor mínimo correspondente à dose de $65 \mathrm{~kg} \mathrm{ha}^{-1}$ de $\mathrm{P}_{2} \mathrm{O}_{5}$. Esse resultado difere do descrito por SILVA et al. (2008), que determinaram redução na concentração do nutriente na mamoneira em resposta ao aumento da adubação fosfatada. O desequilíbrio em relação a outros micronutrientes, como Mn, Cu e Mo, a condição de $\mathrm{pH}$ elevado e o excesso de $\mathrm{P}$ no solo e na planta são condições que predispõem à deficiência de ferro, devido à interação antagônica entre os dois nutrientes (ABREU et al., 2007). Possivelmente, o efeito diverso determinado neste estudo esteja associado ao $\mathrm{pH}$ do solo utilizado $(5,8)$, que se encontra dentro da faixa de maior disponibilidade de ferro $(4,0-6,0)$. Os teores de Fe determinados no experimento foram menores que os relatados por LÉLES (2008), para a 'AL Guarany 2002’, em Latossolo Vermelho distrófico, rico em ferro, do município de Botucatu, São Paulo (SP).
Ambas as cultivares tiveram a produtividade de grãos influenciada pela dose de fósforo, sendo os dados ajustados a modelos quadráticos (Figura 1e). As doses de máxima eficiência técnica (MET) e econômica (MEE) calculadas para a 'Lyra' corresponderam a 90 e $78 \mathrm{~kg} \mathrm{ha}^{-1}$ de P O , respectivamente. Para a 'AL Guarany 2002', as doses de MET e MEE superaram o intervalo de doses de $P$ avaliadas, sendo estimadas em 156 e $134 \mathrm{~kg} \mathrm{ha}^{-1} \mathrm{de}_{2} \mathrm{O}_{5}$. A dose de MEE determinada para ambas as cultivares foi inferior aos $143 \mathrm{~kg} \mathrm{ha}^{-1}$ de $\mathrm{P}_{2} \mathrm{O}_{5}$ determinados por SOUZA et al. (1974), para a 'Campinas', em estudo realizado em Jaboticabal-SP, sob condições climáticas e de cultivo bastante favoráveis. Superaram, porém, os $47 \mathrm{~kg}$ de $\mathrm{P}_{2} \mathrm{O}_{5} \mathrm{ha}^{-1}$ determinados por SOUZA \& NEPTUNE (1976), em estudo em que a resposta da cultura à adubação fosfatada foi alta, atingindo $400 \mathrm{~kg}$ ha $^{-1}$ de $\mathrm{P}_{2} \mathrm{O}_{5}$, o que se explica pelo fato de ter sido realizado num período em que a renda líquida para a aplicação de fertilizantes era negativa. Recentemente, FERRO et al. (2008), em trabalho realizado em Rio Largo, Alagoas (AL), observaram menor resposta à aplicação de P das cultivares BRS 149 'Nordestina' e BRS 188 'Paraguaçu', com dose de MET correspondente a 60kg ha-1 de $\mathrm{P}_{2} \mathrm{O}_{5}$. Os autores associaram a baixa resposta ao nutriente à restrição na disponibilidade de cobre e manganês no solo, uma vez que não houve restrição hídrica durante o período de cultivo. Atribui-se a elevada magnitude de resposta das cultivares 'AL Guarany 2002' e 'Lyra’ à adubação fosfatada ao baixo teor inicial do nutriente no solo $\left(5,9 \mathrm{mg} \mathrm{dm}^{-3}\right)$ e à importância do nutriente para o desempenho produtivo de culturas oleaginosas (FERREIRA et al., 2004). O comportamento observado reforça, ainda, proposição de que o P é o nutriente de maior relevância na produção da mamoneira (SOUZA et al., 1974; NAKAGAWA et al., 1986).

Com os resultados obtidos, conclui-se que os teores foliares de cálcio e boro ('Lyra') na mamoneira diminuem com o aumento da adubação fosfatada. A dose de $\mathrm{P}$ influencia, ainda, o teor de nitrogênio, que aumenta com a aplicação de até $56 \mathrm{~kg} \mathrm{ha}^{-1}$ de $\mathrm{P}_{2} \mathrm{O}_{5}$, e de ferro, que decresce com o uso de até $65 \mathrm{~kg} \mathrm{ha}^{-1}$ de $\mathrm{P}_{2} \mathrm{O}_{5}$ A adubação fosfatada aumenta a produtividade de grãos da mamoneira. Para a 'Lyra', as doses de máxima eficiência técnica e econômica são, respectivamente, de 90 e $78 \mathrm{~kg} \mathrm{ha}^{-1}$ de $\mathrm{P}_{2} \mathrm{O}_{5}$ e, para a 'AL Guarany 2002', são estimadas em 156 e $134 \mathrm{~kg}^{-1}$ he $\mathrm{P}_{2} \mathrm{O}_{5}$.

Ciência Rural, v.40, n.8, ago, 2010. 


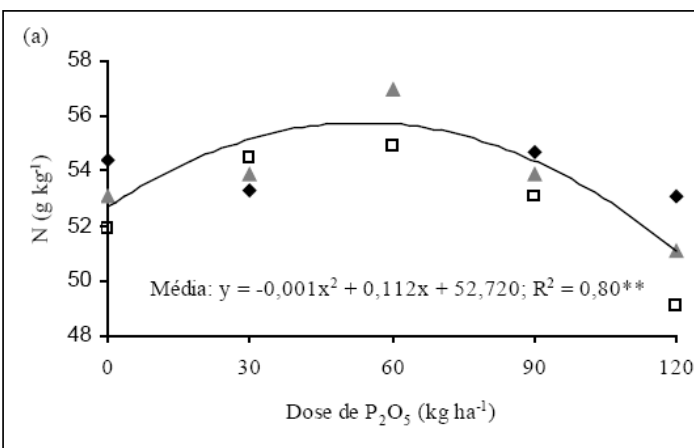

- AL Guarany $\mathbf{L}$ Lyra $\triangle$ Média

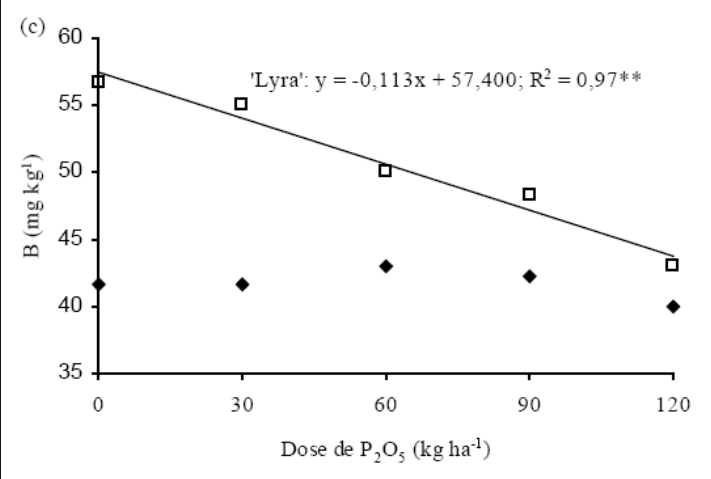

- AL Guarany a Lyra

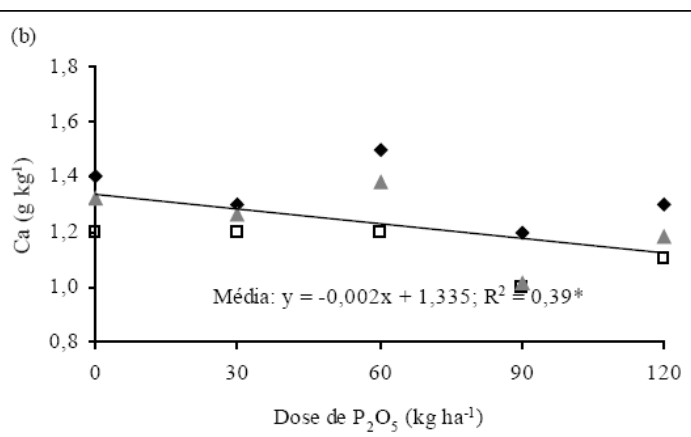

- AL Guarany $\square$ Lyra $\Delta$ Média

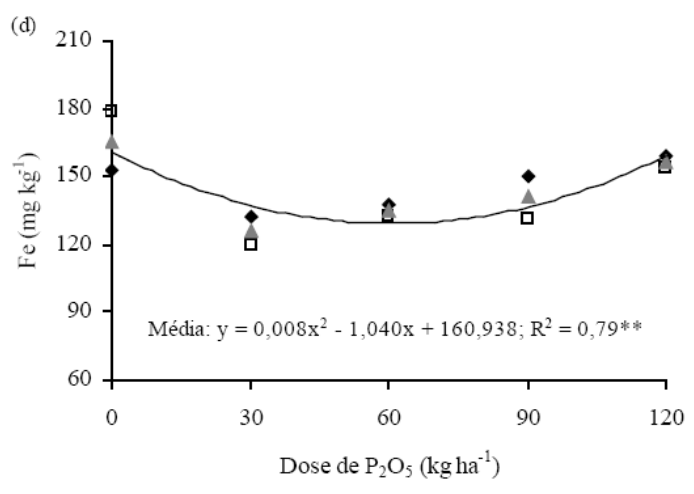

- AL Guarany a Lyra $\triangle$ Média

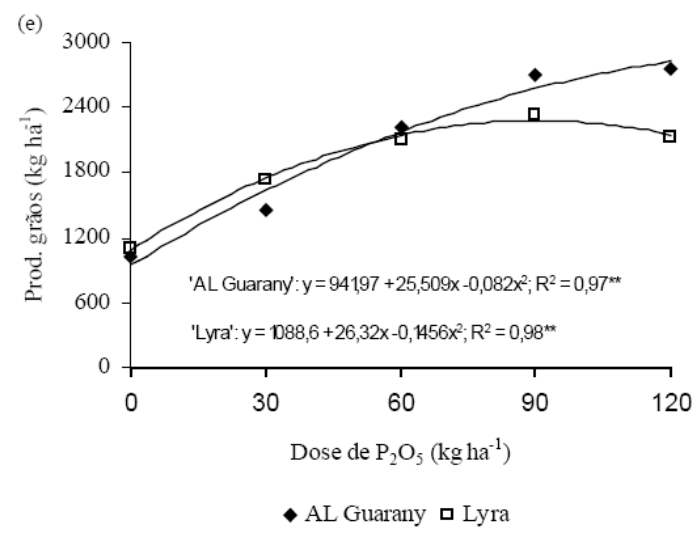

Figura 1 - Teores de nitrogênio (a), cálcio (b), boro (c) e ferro (d) no tecido foliar e produtividade de grãos (e) da mamoneira das cultivares 'AL Guarany 2002' e 'Lyra', em função da dose de fósforo.

\section{REFERÊNCIAS}

ABREU, C.A. de et al. Micronutrientes. In: NOVAIS, R.F. et al. (Eds). Fertilidade do solo. Viçosa: SBCS, 2007. p.645736 .

COMISSÃO DE QUÍMICA E FERTILIDADE DO SOLO DO RS/SC (CQFS RS/SC). Manual de adubação e de calagem para os Estados do Rio Grande do Sul e de Santa Catarina. 10.ed. Porto Alegre: Sociedade Brasileira de Ciência do Solo/ Núcleo Regional Sul, 2004. 400p.
FERREIRA, G.B. et al. A deficiência de fósforo e potássio na mamona (Ricinus communis L.): descrição e efeito sobre o crescimento e a produção da cultura. In: CONGRESSO BRASILEIRO DE MAMONA, 2004, Campina Grande, PB. Anais... Campina Grande: Embrapa Algodão, 2004. 1 CD. Disponível em: <http://www.biodieselbr.com/pdf/mamona/ 076.PDF>. Acesso em: 24 maio, 2010.

FERRO, J.H. de A. et al. Avaliação do crescimento e da produtividade de duas variedades de mamona (Ricinus communis L.) em função da adubação fosfatada no município de Rio Largo 
- AL. In: CONGRESSO BRASILEIRO DE MAMONA, 3., 2008, Salvador, BA. Anais... Salvador: SEAGRI; Embrapa Algodão, 2008. 1 CD. Disponível em: <http://www.cnpa.embrapa.br/ produtos/mamona/publicacoes/cbm3/trabalhos/ FERTILIDADE\%20E\%20ADUBACAO/FA\%2016.pdf >. Acesso em: 24 maio, 2010

FREIRE, C.J. da S. Manual de métodos de análise de tecido vegetal, solo e calcário. 2.ed. Pelotas: Embrapa Clima Temperado, 2001. 201p.

JESCHKE, W.D. et al. Effects of P deficiency on assimilation and transport of nitrate and phosphate in intact plants of castor bean (Ricinus communis L.). Journal of Experimental Botany, v.48, n.306, p.75-91, 1997. Disponível em: <http:// jxb.oxfordjournals.org/cgi/reprint/48/1/75>. Acesso em: 24 maio, 2010. doi: 10.1093/jxb/48.1.75.

LAVRES JUNIOR, J. et al. Deficiências de macronutrientes no crescimento e na produção da mamoneira cultivar Íris. Revista Brasileira de Ciências Agrárias, v.4, n.4, p.405413, 2009. Disponível em: <http://www.agraria.pro.br/sistema/ index.php? journal = a graria \&page $=$ article $\&$ op $=v i$ ew\&path\%5B\%5D=537\&path\%5B\%5D=588>. Acesso em: 24 maio, 2010

LÉLES, E.P. Interação de doses de calcário e zinco na mamoneira. 2008. 66f. Dissertação (Mestrado em Agronomia) - FCA/UNESP, Jaboticabal, SP.

MALAVOLTA, E. et al. Avaliação do estado nutricional das plantas: princípios e aplicações. 2.ed. Piracicaba: POTAFOS, 1997. 319p.

NAKAGAWA, J. et al. Efeitos de doses crescentes de termofosfato na presença e ausência de micronutrientes em dois cultivares de mamoneira (Ricinus communis L.). Científica, v.14, p.55-64, 1986.
NAKAGAWA, J.; NEPTUNE, A.M.L. Marcha de absorção de nitrogênio, fósforo, potássio, cálcio e magnésio na cultura da mamoneira (Ricinus communis L.) cultivar "Campinas". Anais da Escola Superior de Agricultura Luiz de Queiróz, v.28, p.323-337, 1971.

PAULO, E.M. et al. Deficiência de boro em mamona Bragantia, v.48, p.241-247, 1989. Disponível em: <http:// www.iac.sp.gov.br/bragantia/volume/4802/480210.pdf >. Acesso em: 24 maio, 2010.

SANTOS, H.G. dos et al. (Ed.). Sistema brasileiro de classificação de solos. 2.ed. Rio de Janeiro: Embrapa Solos, 2006. 306p.

SAVY FILHO, A. Mamona: tecnologia agrícola. Campinas: EMOPI, 2005. 105p.

SILVA, D.F. et al. Estado nutricional e acúmulo de nutrientes em mamoneira submetidas a diferentes doses de fósforo. In: CONGRESSO BRASILEIRO DE MAMONA, 3., 2008, Salvador, BA. Anais... Salvador: SEAGRI; Embrapa Algodão, 2008. 1 CD. Disponível em: <http://www.cnpa.embrapa.br/produtos/ $\mathrm{m}$ a mon a / p u bl i c a c o e s / c b m 3/ trabalhos/ FERTILIDADE\%20E\%20ADUBACAO/FA\%2015.pdf>. Acesso em: 24 maio, 2010

SOUZA, E.A.; NATALE, W. Efeito do boro e zinco na cultura da mamoneira. Científica, v.2, p.327-333, 1997.

SOUZA, E.A. et al. Adubação fosfatada na cultura da mamoneira (Ricinus communis (L.) cv. Guarani). Científica, v.3, p.1928, 1985.

SOUZA, E.A. et al. Efeitos da fertilização nitrogenada, fosfatada e potássica na produção da mamoneira (Ricinus communis L.). Científica, v.2, p.62-168, 1974.

SOUZA, E.A.; NEPTUNE, A.M.L. Resposta da cultura de Ricinus communis L. à adubação e calagem. Científica, v.4, p.274-281, 1976 